\title{
Comprovação do efeito antioxidante de plantas medicinais utilizadas no tratamento do Diabetes mellitus em animais: artigo de atualização
}

\author{
DALLAQUA, B.; DAMASCENO, D.C.* \\ Laboratório de Pesquisa Experimental de Ginecologia e Obstetrícia, Departamento de Ginecologia e Obstetrícia, \\ Faculdade de Medicina de Botucatu - Unesp, Distrito de Rubião Júnior, s/n, CEP: 18603-970, Botucatu-Brasil \\ *damasceno@fmb.unesp.br
}

\begin{abstract}
RESUMO: Diabetes mellitus (DM) é uma síndrome de etiologia múltipla caracterizada por hiperglicemia crônica. Esta hiperglicemia induz o aumento na produção de espécies reativas de oxigênio (ERO) e diminuição das defesas antioxidantes. Devido às complicações causadas pelo diabete, muitos indivíduos optam por terapias alternativas à base de plantas medicinais para amenizar seus efeitos. Sendo assim, nesta revisão de literatura, foram analisados e descritos diversos trabalhos experimentais com a utilização de animais diabéticos para comprovar os efeitos antioxidantes de algumas dessas plantas e verificar se os títulos e resumos disponibilizados nos artigos são compatíveis aos objetivos de nossa busca.
\end{abstract}

Palavras-chave: diabete, plantas, antioxidantes, ratos

\begin{abstract}
Evidence of the antioxidant effect of medicinal plants used in the treatment of Diabetes mellitus in animals: an update. Diabetes mellitus (DM) is a syndrome of multiple etiology characterized by chronic hyperglycemia. This hyperglycemia induces increased production of reactive oxygen species (ROS) and decreased antioxidant defenses. Due to complications caused by diabetes, a large number of people have chosen medicinal plant-based alternative therapies to alleviate its effects. Thus, in this literature review, several experimental studies with the use of diabetic animals were analyzed to demonstrate the antioxidant effects of these plants and to verify if the titles and abstracts provided in the papers are compatible with the aims of our search.
\end{abstract}

Keywords: diabetes, plant, antioxidant, rats

Diversas drogas são utilizadas para o controle dos níveis glicêmicos em portadores de Diabetes mellitus (DM), entretanto o perfeito controle é raramente alcançado (Damasceno et al., 2004; Takaku et al., 2006). DM é uma desordem crônica causada pela falta de produção de insulina pelas células beta ( $\beta$ )-pancreáticas ou pelo defeito nos receptores de insulina nas células-alvo, resultando em doença metabólica hiperglicêmica (American Diabetes Association - ADA, 2009). DM é situação clínica frequente, acomete em torno de $7 \%$ da população. Cerca de $50 \%$ dos portadores de diabete desconhecem o diagnóstico (Takaku et al., 2006; ADA, 2009). A deficiênca de insulina resulta em níveis elevados de glicose sanguínea, que, por sua vez, pode causar danos em vários sistemas do organismo (Leahy, 2005), alterando o metabolismo de carboidratos, lipídios e proteínas (ADA, 2009). A síndrome diabética pode apresentar-se com sintomas característicos, tais como sede, poliúria, visão turva, perda de peso e polifagia e, nas formas mais graves, pode ocorrer cetoacidose, que, na ausência de tratamento eficaz, ocasiona hálito acético, coma e morte (Davidson, 2001).

DM pode ser classificado em tipo 1 (DM1), tipo 2 (DM2) e gestacional. DM1 é a forma auto-imune, resulta da destruição das células $\beta$-pancreáticas por mecanismo mediado por células. No DM2, os indivíduos afetados apresentam resistência à insulina, em combinação com deficiência relativa (não absoluta) da secreção de insulina (Davidson, 2001; ADA, 2009). DM gestacional é caracterizado pelo quadro de intolerância à glicose, com primeira identificação na gravidez e pode persistir após o parto evoluindo para

Recebido para publicação em 10/12/2009

Aceito para publicação em 05/01/2011

Rev. Bras. PI. Med., Botucatu, v.13, n.3, p.367-373, 2011. 
DM2 (Buchanan et al., 2005; 2007).

A hiperglicemia crônica no diabete provoca glicação de proteínas que, por sua vez, leva a complicações secundárias que afetam olhos, rins, nervos e artérias (Sharma \& Misra, 1993). A síndrome diabética está relacionada à formação de radicais livres, que são espécies reativas de oxigênio (ERO) formadas através da redução de um ou dois elétrons de oxigênio $\left(\mathrm{O}_{2}\right)$ (Eriksson \& Born, 1993; Reece et al., 1998; Zhao \& Reece, 2005; Damasceno et al., 2006; Cimen, 2008). Em situações de estresse, nosso organismo gera desequilíbrio entre os agentes oxidantes e antioxidantes para se proteger, resultando no quadro de estresse oxidativo (Halliwell \& Gutteridge, 1999; Damasceno et al., 2002).

A fonte primária para formação de ERO é o aumento do influxo de piruvato e oxigênio na mitocôndria e aumento na produção de ERO (principalmente superóxido) em processos oxidativos na cadeia de transporte de elétrons (Yang et al., 1997). O radical superóxido passa para outros compartimentos da mitocôndria e citosol promovendo a formação de peróxido de hidrogênio e radical hidroxil que causará alterações na mitocôndria (Yang et al., 1995), acarretando lipoperoxidação (Wentzel et al., 1999) e danos no DNA (Lee et al., 1999).

A primeira linha de defesa contra os danos oxidativos são os antioxidantes endógenos enzimáticos, o superóxido dismutase (SOD), a catalase e o sistema de glutationas, dentre elas, glutationa redutase (GSH-Rd) e glutationa peroxidase (GSH-Px). Há também os antioxidantes nãoenzimáticos que são lipossolúveis (tocoferois, carotenos, quinonas e bilirrubinas) e hidrossolúveis (ácido ascórbico, ácido úrico e proteínas ligadas a metais) (Halliwell \& Gutteridge, 1999; Damasceno et al., 2002).

Embora a produção de ERO sob condições hiperglicêmicas não seja demonstrada diretamente, 0 estresse oxidativo é identificado por métodos indiretos, como pela peroxidação fosfolipídica, redução nos níveis intracelulares de enzimas antioxidantes (SOD, Catalase e GSH-Px) e depleção da capacidade antioxidante do meio hiperglicêmico (Trocino et al., 1995; Cederberg \& Eriksson, 1997). ERO pode ser prejudicial para as células porque reagem com ácidos graxos insaturados que são encontrados nas membranas plasmáticas, produzindo peróxidos lipídicos que leva à diminuição da fluidez da membrana (Kaplan et al., 1995) e à formação de aldeídos reativos, que podem reagir com macromoléculas (Gutteridg \& Halliwell, 1990; Mattson, 1998). ERO também pode reagir diretamente com proteínas, resultando em ligações cruzadas de colágeno com o DNA, causando danos em suas bases e açúcares (Imlay \& Linn 1988; Elgawish et al., 1996).

Estudos realizados em humanos para explorar os mecanismos responsáveis pelas alterações causadas pelo diabete são limitados não somente por razões éticas, mas também pela multiplicidade de variáveis não controladas, como hábitos alimentares, fatores socioeconômicos, nutrição e fatores genéticos. Desta forma, existe a necessidade de reproduzir modelos experimentais para mimetizar a síndrome diabética (López-Soldado \& Herrera, 2003; Souza et al., 2009; Sinzato et al., 2009). Para isso, são utilizadas drogas $\beta$-citotóxicas como streptozotocin (STZ) e aloxana. Aaloxana induz a formação de ERO resultando em necrose das células $\beta$-pancreáticas (Lenzel, 2008), sendo assim, esta droga além de lesar o pâncreas prejudica outros tecidos, pois não apresenta especificidade somente às células $\beta$-pancreáticas. $A$ aloxana vem sendo menos empregada pelos pesquisadores em função de efeitos adversos e a pouca margem de segurança entre a dose letal e a dose eficaz para obtenção do quadro diabético (lessi et al., 2007). Já, o STZ inibe a síntese de pró-insulina nas células $\beta$-pancreáticas e também induz alquilação do DNA, resultando em fragmentação e consequente morte da célula $\beta$ (Lenzel, 2008). Desta forma, STZ não causa dano em outros tecidos do organismo (lessi et al., 2007), com isso mostra ser mais eficaz para indução do modelo de diabete experimental.

Frente aos conhecimentos adquiridos sobre a formação de ERO e os antioxidantes presentes em nosso organismo para se defender contra os insultos oxidativos (estresse oxidativo exacerbado), diversos pesquisadores têm investigado substâncias com efeito antioxidante.

Atualmente, mais de 800 tipos de plantas são utilizados no tratamento do DM (Saxena et al., 2004) e a maioria destas apresentam amplo espectro na clínica. Sendo assim, há necessidade em se explorar o campo da fitomedicina para fornecer terapias alternativas para tratamento da síndrome diabética (Yeh et al., 2003; Suba et al., 2004).

Apesar do grande interesse no desenvolvimento de novas drogas para prevenir complicações associadas a esta doença, a comunidade científica tem interesse em avaliar o estado do produto natural isolado em estudos experimentais, sendo que alguns deles já foram testados em seres humanos (Liu et al., 2004; Vuksan \& Sievenpiper, 2005; Johnson et al., 2006; Jung et al., 2006; Matsui et al., 2006). Suplementos naturais são amplamente utilizados em todo o mundo para tratar o diabete (Liu et al., 2004). No entanto, a maioria dos produtos naturais é consumida de forma indiscriminada e grande parte deles não foi testada ou não teve efeito confirmado.

Desta forma, este estudo tem como objetivo apresentar uma atualização bibliográfica das plantas medicinais utilizadas para o tratamento do DM e que apresentaram atividade antioxidante. 
Foi realizada revisão de literatura referente ao período de 1992 a 2009 (17 anos) no site do database do National Center of Biotechnology Information (NCBIPUB MED). Para busca dos artigos, foram empregados os unitermos Plant and antioxidant and diabetes and rats e foram encontrados 313 artigos.

Após leitura e análise dos títulos e resumos, do total de 313 artigos encontrados, 134 (42,8\%) eram coerentes quanto ao título e resultados; 87 (27,8\%) apresentaram título e resultados não adequados à investigação; 53 (16,9\%) tinham título adequado e resultados não pertinentes e 39 (12,5\%) apresentaram título não compatível à pesquisa e resultados coerentes.

A Tabela 1 mostra os resultados com relação às plantas mais conhecidas que apresentaram atividade antioxidante confirmada cientificamente dentre os artigos analisados.

TABELA 1. Plantas que apresentam efeito antioxidante.

\begin{tabular}{|c|c|c|c|c|c|}
\hline Planta & Nome Popular & $\begin{array}{c}\text { Parte } \\
\text { utiliza da }\end{array}$ & Interv enção & Dose & Referência \\
\hline Aegle marmelos & - & Fruto & Extrato aquoso & 125 e $250 \mathrm{mg} \mathrm{Kg}^{-1}$ & $\begin{array}{l}\text { Kamalakknnan \& } \\
\text { Stanley (2003) }\end{array}$ \\
\hline Allium cepa & Cebola & - & $\begin{array}{l}\text { Solução de } \\
\text { Allium cepa }\end{array}$ & $0.4 \mathrm{~g} \mathrm{~A}$. ce pa/rato & Campos et al. (2003) \\
\hline Allium sativum & Alho & - & $\begin{array}{c}\text { Extrato } \\
\text { meta nólico }\end{array}$ & 250 e $500 \mathrm{mg} \mathrm{Kg}^{-1}$ & Rajani et al. (2008) \\
\hline Aloe vera & Aloe vera & Folhas & Extrato gel & $300 \mathrm{mg} \mathrm{Kg}^{-1}$ & $\begin{array}{c}\text { Rajase karan et al. } \\
(2005)\end{array}$ \\
\hline $\begin{array}{l}\text { Andrograph is } \\
\text { paniculata }\end{array}$ & - & Folh as & Extrato aquoso & $400 \mathrm{mg} \mathrm{Kg}^{-1}$ & $\begin{array}{l}\text { Dandu \& Inam dar } \\
\qquad(2009)\end{array}$ \\
\hline Annona squamosa & Pinha ou Ateira & Folhas & - & - & Panda \& Kar (2007) \\
\hline Bauhinia forficata & Pata de vaca & Folhas & Extrato aquoso & $500,600 \underset{\mathrm{Kg}^{-1}}{\mathrm{e} 1000 \mathrm{mg}}$ & Volpa to et al. (2008) \\
\hline Brassica oleracea & Couve & Flores & $\begin{array}{c}\text { Frações de } \\
\text { MeOH, } \\
\mathrm{CH} 2 \mathrm{Cl} 2, \mathrm{BuOH}\end{array}$ & 100 e $200 \mathrm{mg} \mathrm{Kg}^{-1}$ & Cho etal. (2006) \\
\hline Camellia sinensis & Chá verde & Folhas & Extrato aquoso & $500 \mathrm{mg} \mathrm{Kg}^{-1}$ & Sabu et al. (2002) \\
\hline Case aria esculenta & - & Raiz & Extrato aquoso & 200 e $300 \mathrm{mg} \mathrm{Kg}^{-1}$ & $\begin{array}{l}\text { Prakasam et al. } \\
\qquad(2003)\end{array}$ \\
\hline Cassia fistula & Chuva de ouro & Flores & Extrato aquoso & $20 \mathrm{~g} \mathrm{Kg}^{-1}$ & $\begin{array}{l}\text { Manonmani et al. } \\
(2005)\end{array}$ \\
\hline Commelin a communis & - & - & Extrato e pó & - & Shibano et al. (2008) \\
\hline Coccinia indica & - & Folhas & $\begin{array}{l}\text { Extrato } \\
\text { etanólico }\end{array}$ & $200 \mathrm{mg} \mathrm{Kg}^{-1}$ & $\begin{array}{c}\text { Venkateswaran \& } \\
\text { Pari (2003) }\end{array}$ \\
\hline Gymnema montanum & - & Folhas & $\begin{array}{l}\text { Extrato } \\
\text { alcoólico }\end{array}$ & $200 \mathrm{mg} \mathrm{Kg}^{-1}$ & $\begin{array}{l}\text { A nanth an et al. } \\
\qquad(2003)\end{array}$ \\
\hline Ginkgo biloba & Ginkgo biloba & Folhas & Extrato aquoso & $200 \mathrm{mg} \mathrm{Kg}^{-1}$ & Rudge et al. (2007) \\
\hline $\begin{array}{l}\text { Gongronema } \\
\text { latifolium }\end{array}$ & - & Folhas & $\begin{array}{l}\text { Extrato a quoso } \\
\text { e etanólico }\end{array}$ & $100 \mathrm{mg} \mathrm{Kg}^{-1}$ & $\begin{array}{l}\text { Ugo chukwu \& } \\
\text { Coboume (2003) }\end{array}$ \\
\hline Hibiscus sabdariffa & $\begin{array}{l}\text { Q uiabo-roxo; } \\
\text { vinag re ira; } \\
\text { caruru -a zedo }\end{array}$ & Flores & $\begin{array}{c}\text { Ext ra to } \\
\text { Etanólico }\end{array}$ & 100 e $200 \mathrm{mg} \mathrm{Kg}^{-1}$ & $\begin{array}{l}\text { Farombi \& lge } \\
(2007)\end{array}$ \\
\hline Lam in aria japon ica & Algas Kombu & Talo & Extrato a quoso & $100 \mathrm{mg} \mathrm{Kg}^{-1}$ & Jin et al. (2004) \\
\hline Matricaria c hamomilla & Camomila & Folhas & $\begin{array}{c}\text { Ext rato } \\
\text { Etanólico }\end{array}$ & 20,50 e $100 \mathrm{mg} \mathrm{Kg}^{-}$ & Cemek et al. (2008) \\
\hline $\begin{array}{l}\text { Morus bo mbycis } \\
\text { koidzumi }\end{array}$ & - & Raiz & - & $200-800 \mathrm{mg} \mathrm{Kg}^{-1}$ & Heo et al. (2007) \\
\hline Morus in dica & Amora & Folhas & Pó das folhas & $25 \%$ & $\begin{array}{l}\text { Andallu \& } \\
\text { Varadacharyulu } \\
(2003)\end{array}$ \\
\hline
\end{tabular}

Rev. Bras. Pl. Med., Botucatu, v.13, n.3, p.367-373, 2011. 
TABELA 1. Plantas que apresentam efeito antioxidante.

... continuação

\begin{tabular}{|c|c|c|c|c|c|}
\hline Planta & Nome Popular & $\begin{array}{c}\text { Parte } \\
\text { utilizada }\end{array}$ & Intervenção & Dose & Referência \\
\hline Panax ginseng & Ginseng & Folhas & Extrato aquoso & 40 e $200 \mathrm{mg} \mathrm{Kg}^{-1}$ & Jung et al. (2005) \\
\hline Phaseolus vulgaris & $\begin{array}{l}\text { Feijão-base, } \\
\text { feijão-comum, } \\
\text { feijoeiro }\end{array}$ & Vagens & Extrato aquoso & $200 \mathrm{mg} \mathrm{Kg}^{-1}$ & $\begin{array}{l}\text { Venkateswaran et } \\
\text { al. (2002) }\end{array}$ \\
\hline Quercetin allievates & - & - & - & 50 e $80 \mathrm{mg} \mathrm{Kg}^{-1}$ & $\begin{array}{l}\text { Mahesh \& Menon } \\
(2004)\end{array}$ \\
\hline Scoparia dulcis & $\begin{array}{l}\text { Vassourinha- } \\
\text { doce }\end{array}$ & $\begin{array}{l}\text { Toda a } \\
\text { planta }\end{array}$ & Extrato aquoso & $200 \mathrm{mg} \mathrm{Kg}^{-1}$ & Pari \& Latha (2004) \\
\hline Syzigium cumini & $\begin{array}{c}\text { Jambolão, } \\
\text { Jamelão, Jalão }\end{array}$ & Sementes & Extrato aquoso & 2,5 e $5,0 \mathrm{~g} \mathrm{Kg}^{-1}$ & Prince et al. (1998) \\
\hline Tinospora cordifolia & - & Raiz & Extrato alcoólico & $100 \mathrm{mg} \mathrm{Kg}^{-1}$ & Prince et al. (2004) \\
\hline $\begin{array}{l}\text { Trigonella foenum } \\
\text { graecum }\end{array}$ & Feno-grego & Sementes & Extrato aquoso & $2 \mathrm{~g} \mathrm{Kg}^{-1}$ & $\begin{array}{l}\text { Anuradha \& } \\
\text { Ravikumar (2001) }\end{array}$ \\
\hline
\end{tabular}

Frente esta revisão de literatura, pode ser verificado que diferentes formas de preparo dos produtos naturais podem levar a efeitos similares como mostrado nos trabalhos realizados com extrato metanólico (250 e $500 \mathrm{mg} \mathrm{Kg}^{-1}$ ) (Rajani et al., 2008), sumo $(0,4 \mathrm{~g} 100 \mathrm{~g})$ (El-Demerdash et al., 2005) e o óleo de Allium sativum (10 mg Kg-1) (Anwar \& Meki, 2003), que observaram elevação nas atividades das enzimas antioxidantes.

Da mesma forma, estudos realizados com Allium cepa (cebola), utilizando diferentes preparações, mostraram que animais diabéticos tratados com o sumo (El-Demerdash et al., 2005) apresentam diminuição na concentração de TBARS e aumento das atividades das enzimas antioxidantes no plasma, fígado, cérebro e rim. Ao serem tratados com o composto isolado de Allium cepa (Kumari \& Augusti, 2002) também se observou aumento de enzimas antioxidantes, mas somente no plasma.

Camellia sinensis, conhecida popularmente como chá verde, vem despertando interesse em todo mundo. Diferentes artigos de diversos países confirmaram seu potencial antioxidante, dentre eles Juskiewicz et al. (2008), pesquisadores da Polônia, observaram aumento nas atividades de GSH-Px e de SOD no soro e diminuição na concentração do marcador de lipoperoxidação (TBARS) no rim de ratos diabéticos. Babu et al. (2006), da Índia, trataram ratos diabéticos e comprovaram redução de TBARS e aumento na atividade de GSH no coração. Nos EUA, Mustata et al. (2005) evidenciaram aumento nos níveis de GSH e diminuição de hidroperóxidos no soro de animais diabéticos. Sabu et al. (2002), pesquisadores da Índia, também mostraram que várias doses de Camellia sinensis aumentaram as atividades das enzimas antioxidantes SOD e GSH e redução das concentrações dos marcadores de lipoperoxidação em animais diabéticos.
Pesquisas realizadas com diferentes tipos de extratos de Andrographis paniculata mostraram que o extrato etanólico (Zhang \& Tan, 2000) e o extrato aquoso (Dandu \& Inamdar, 2009) das folhas desta planta apresentaram aumento nas atividades de SOD e CAT, mas não apresentaram efeito significativo sobre a atividade de GSH-Px em ratos diabéticos.

O extrato aquoso de Annona squamosa exerceu efeito antioxidante em animais diabéticos onde observaram aumento das atividades de CAT, SOD e GSH e diminuição nas concentrações de marcadores de lipoperoxidação (Panda \& Kar, 2007; Gupta et al., 2008).

Experimentos realizados com diferentes preparações de Aegle marmelos para verificar o possível efeito antioxidante desta planta em diferentes tecidos evidenciaram que o extrato metanólico diminuiu a concentração de TBARS no soro e fígado e aumentou as atividades das enzimas antioxidantes CAT e GSH-Px no fígado de animais diabéticos (Sabu \& Kuttan, 2004). Kamalakkannan \& Stanley (2003), ao tratarem animais diabéticos com extrato aquoso da mesma planta, mostraram aumento das mesmas enzimas antioxidantes no pâncreas e coração.

Embora, muitos trabalhos sejam realizados para verificar os efeitos antioxidantes de plantas medicinais em animais de laboratório, diversos pesquisadores não concluem os mesmos efeitos nos animais em comparação aos obtidos na população em geral. Isto pode ser devido à diferença de sensibilidade existente entre as espécies, tipo de preparo com as plantas, dose e via de administração utilizadas.

Além disso, cabe ressaltar que os resumos não descrevem de forma satisfatória os resultados obtidos para esclarecimento do artigo como um todo, dificultando a busca por artigos coerentes à pesquisa. Desta forma, este artigo de atualização confirmou a

Rev. Bras. PI. Med., Botucatu, v.13, n.3, p.367-373, 2011. 
necessidade de se redigir títulos e resumos adequados ao estudo para viabilizar a busca de informações.

\section{REFERÊNCIA}

AMERICAN DIABETES ASSOCIATION (ADA). Diagnosis and classification of diabete mellitus. Diabetes Care, v.32, suppl.1, p.S62-7, 2009. Disponível em: <http:// care.diabetesjournals.org/cgi/reprint/32/Supplement_1/ S62>. Acesso em: 30 mar. 2009.

ANANTHAN, R. et al. Antidiabetic effect of Gymnema montanum leaves: effect on lipid peroxidation induced oxidative stress in experimental diabetes. Pharmacology Research, v.48, n.6, p.551-6, 2003.

ANDALLU, B.; VARADACHARYULU, N.C.H. Antioxidant role of mulberry (Morus indica) leaves in streptozotocindiabetic rats. Clinica Chimica Acta, v.338, n.1-2, p.3-10, 2003.

ANURADHA, C.V.; RAVIKUMAR, P. Restoration on tissue antioxidants by fenugreek seeds (Trigonella foenum graecum) in alloxan-diabetic rats. Indian Journal of Physiology \& Pharmacology, v.45, n.4, p.408-20, 2001. ANWAR, M.M.; MEKI, A.R. Oxidative stress in streptozotocin-induced diabetic rats: effects of garlic oil and melatonin. Comparative Biochemistry and Physiology Part A: Molecular \& Integrative Physiology, v.135, n.4, p.539-47, 2003.

BABU, P.V.; SABITHA, K.E.; SHYAMALADEVI, C.S. Therapeutic effect of green tea extract on oxidative stress in aorta and heart of streptozotocin diabetic rats. Chemico-Biological Interactions, v.162, n.2, p.114-20, 2006.

BUCHANAN, T.A.; XIANG, A.H. Gestational diabetes mellitus. The Journal of Clinical Investigation, v.115, n.3, p.485-91, 2005.

BUCHANAN, T.A. et al. What is gestational diabetes? Diabetes Care, v.30, n.2, p.S105-11, 2007.

CAMPOS, K.E. et al. Hypoglycaemic and antioxidant effects of onion, Allium cepa: dietary onion addition, antioxidant activity and hypoglycaemic effects on diabetic rats. International Journal of Food Science and Nutrition, v.54, n.3, p.241-6, 2003.

CEDERBERG, J.; ERIKSSON, U.J. Decreased catalase activity in malformation-prone embryos of diabetic rats. Teratology, v.56, p.350-7, 1997.

CEMEK, M. et al. Antihyperglycemic and antioxidative potential of Matricaria chamomilla in streptozotocininduced diabetic rats. Nature Medicine, v.62, n.3, p.28493, 2008.

$\mathrm{CHO}$, E.J. et al. Protective effects of broccoli (Brassica oleracea) against oxidative damage in vitro and in vivo. Journal of Nutritional Science and Vitaminology, v.52, n.6, p.437-44, 2006.

CIMEN, M.Y. Free radical metabolism in human erythrocytes. Clinica Chimica Acta, v.390, n.1, p.1-11, 2008.

DAMASCENO, D.C. et al. Radicais livres, estresse oxidativo e diabete. Diabetes Clínica, v.5, p.355-61, 2002. DAMASCENO, D.C. et al. Effect of Bauhinia forficata extract in diabetic pregnant rats: maternal repercussions. Phytomedicine, v.11, n.2-3, p.196-201, 2004.
DAMASCENO, D.C.; SOUZA, M.S.S.; RUDGE, M.V.C. Diabetes e malformações congênitas - estudos em animais e humanos. Metabólica, v.8, n.6, p.57-62, 2006. DANDU, A.M.; INAMDAR, N.M. Evaluation of beneficial effects of antioxidant properties of aqueous leaf extract of Andrographis paniculata in STZ-induced diabetes. Pakistan Journal of Pharmaceutical Science, v.22, n.1, p.49-52, 2009.

DAVIDSON, M.B. Diabete mellitus diagnóstico e tratamento. 4.ed. Rio de Janeiro: Revinter, 2001. 389p. EL-DEMERDASH, F.M.; YOUSEF, M.I.; EL-NAGA, N.I. Biochemical study on the hypoglycemic effects of onion and garlic in alloxan-induced diabetic rats. Food and Chemical Toxicology, v.43, n.1, p.57-63, 2005.

ELGAWISH, A. et al. Involvement of hydrogen peroxide in collagen cross-linking by high glucose in vitro and in vivo. Journal of Biological Chemistry, v.271, n.22, p.12964-71, 1996.

ERIKSSON, U.J.; BORN, L.A. Diabetes and embryonic malformations. Role of substrate-induced free-oxygen radical production for dysmorphogenesis in cultured rat embryos. Diabetes, v.42, n.3, p.411-9, 1993.

FAROMBI, E.O.; IGE, O.O. Hypolipidemic and antioxidant effects of ethanolic extract from dried calyx of Hibiscus sabdariffa in alloxan-induced diabetic rats. Fundamental \& Clinical Pharmacology, v.21, n.6, p.601-9, 2007.

GUPTA, R.K. et al. In vivo evaluation of anti-oxidant and anti-lipidimic potential of Annona squamosa aqueous extract in Type 2 diabetic models. Journal of Ethnopharmacology, v.118, n.1, p.21-5, 2008.

GUTTERIDGE, J.M.; HALLIWELL, B. The measurement and mechanism of lipid peroxidation in biological systems. Trends in Biochemical Science, v.15, p.12935, 1990.

HALLIWELL, B.; GUTTERIDGE, J.M.C. Free radicals in biology and medicine. 3.ed. United Kingdom: Oxford University Press, 1999. 851p.

HEO, S.I. et al. Antidiabetic properties of 2,5-dihydroxy4,3'-di(beta-D-glucopyranosyloxy)-trans-stilbene from mulberry (Morus bombycis koidzumi) root in streptozotocin-induced diabetic rats. Journal of Medical Food, v.10, n.4, p.602-7, 2007.

IESSI, I.L. et al. Drogas beta-citotóxicas: Streptozotocin x Alozana. Diabetes clínica, v.11, n.5, p.435-46, 2007.

IMLAY, J.A.; LINN, S. DNA damage and oxygen radical toxicity. Science, v.240, p.1302-9, 1988.

JIN, D.Q. et al. Preventive effects of Laminaria japonica aqueous extract on the oxidative stress and xanthine oxidase activity in streptozotocin-induced diabetic rat liver. Biological \& Pharmaceutical Bulletin, v.27, n.7, p.103740, 2004.

JOHNSON, L. et al. Use of herbal remedies by diabetic Hispanic women in the southwestern United States. Phytotherapy Research, v.20, p.250-5, 2006.

JUNG, C.H. et al. Effects of wild ginseng (Panax ginseng C.A. Meyer) leaves on lipid peroxidation levels and antioxidant enzyme activities in streptozotocin diabetic rats. Journal of Ethnopharmacology, v.98, n.3, p.24550, 2005.

JUNG, M. et al. Antidiabetic agents from medicinal plants. Current Medicinal Chemistry, v.13, p.1203-18, 2006.

JUSKIEWICZ, J. et al. Extract of green tea leaves partially attenuates streptozotocin-induced changes in antioxidant

Rev. Bras. PI. Med., Botucatu, v.13, n.3, p.367-373, 2011. 
status and gastrointestinal functioning in rats. Nutrition Research, v.28, n.3, p.343-9, 2008.

KAMALAKKANNAN, N.; STANELY, M.P.P. Effect of Aegle marmelos correa fruit extract on tissue antioxidants in streptozotocin diabetic rats. Indian Journal of Experimental Biology, v.41, n.11, p.1285-8, 2003.

KAPLAN, P. et al. Change in fluidity of brain endoplasmic reticulum membranes by oxygen free radicals: a protective effect of stobadine, alpha-tocopherol acetate, and butylated hydroxytoluene. Neurochemical Research, v.20, p.815-20, 1995.

KUMARI, K.; AUGUSTI, K.T. Antidiabetic and antioxidant effects of S-methyl cysteine sulfoxide isolated from onions (Allium cepa Linn) as compared to standard drugs in alloxan diabetic rats. Indian Jornal of Experimental Biology, v.40, n.9, p.1005-9, 2002.

LEAHY, J.L. Pathogenesis of type 2 diabetes mellitus. Archives of Medicine Research, v.36, p.197-209, 2005.

LEE, A.T.; REIS, D.; ERIKSSON, U.J. Hyperglycemia induced embryonic dysmorphogenesis correlates with genomic DNA mutation frequency in vitro and in vivo. Diabetes, v.48, p.371-6, 1999.

LENZEL, S. The mechanisms of alloxan and streptozotocininduced diabetes. Diabetologia, v.51, p.216-26, 2008.

LIU, J.P. et al. Chinese herbal medicines for type-2 diabetes mellitus. Cochrane Database of Systematic Reviews, v.3, p.CD003642, 2004.

LÓPEZ-SOLDADO, I.; HERRERA, E. Different diabetogenic response to moderate doses of streptozotocin in pregnant rats, and its long-term consequences in the offspring. Experimental Diabetes Research, v.4, n.2, p.107-18, 2003. MAHESH, T.; MENON, V.P. Quercetin allievates oxidative stress in streptozotocin-induced diabetic rats. Phytotherapy Research, v.18, n.2, p.123-7, 2004.

MANONMANI, G. et al. Antioxidant activity of Cassia fistula flowers in alloxan induced diabetic rats. Journal of Ethnopharmacology, v.97, n.1, p.39-42, 2005.

MATSUI, T. et al. Antihyperglycemic potential of natural products. Mini Reviews in Medicinal Chemistry, v.6, p.10920, 2006.

MATTSON, M.P. Modification of ion homeostasis by lipid peroxidation: roles in neuronal degeneration and adaptive plasticity. Trends in Neurosciences, v.21, p.53-7, 1998.

MUSTATA, G.T. et al. Paradoxical effects of green tea (Camellia sinensis) and antioxidant vitamins in diabetic rats: improved retinopathy and renal mitochondrial defects but deterioration of collagen matrix glycoxidation and crosslinking. Diabetes, v.54, n.2, p.517-26, 2005.

PANDA, S.; KAR, A. Antidiabetic and antioxidative effects of Annona squamosa leaves are possibly mediated through quercetin-3-O-glucoside. Biofactors, v.31, n.3-4, p.20110, 2007.

PARI, L.; LATHA, M. Protective role of Scoparia dulcis plant extract on brain antioxidant status and lipidperoxidation in STZ diabetic male Wistar rats. BMC Complementary and Alternative Medicine, v.4, n.16, p.1-8, 2004.

PRAKASAM, A. et al. Erythrocyte redox status in streptozotocin diabetic rats: effect of Casearia esculenta root extract. Pharmazie, v.58, n.12, p.920-4, 2003.

PRINCE, P.S.; MENON, V.P.; PARI, L. Hypoglycaemic activity of Syzigium cumini seeds: effect on lipid peroxidation in alloxan diabetic rats. Journal of Ethnopharmacology, v.61, n.1, p.1-7, 1998.
PRINCE, P.S.; KAMALAKKANNAN, N.; MENON, V.P. Restoration of antioxidants by ethanolic Tinospora cordifolia in alloxan-induced diabetic Wistar rats. Acta Poloniae Pharmaceutica, v.61, n.4, p.283-7, 2004.

RAJANI, K.V.; UMA, M.R.P.; RAJU, T.N. Attenuation of streptozotocin-induced oxidative stress in hepatic and intestinal tissues of Wistar rat by methanolic-garlic extract. Acta Diabetology, v.45, n.4, p.243-51, 2008.

RAJASEKARAN, S.; SIVAGNANAM, K.; SUBRAMANIAN, S. Antioxidant effect of Aloe vera gel extract in streptozotocininduced diabetes in rats. Pharmacolology Reproduction, v.57, n.1, p.90-6, 2005.

REECE, E.A. et al. The role of free radicals and membrane lipids in diabetes-induced congenital malformations. Journal of the Society for Gynecologic Investigation, v.5, p.178-87, 1998.

RUDGE, M.V.C. et al. Effect of Ginkgo biloba on the reproductive outcome and oxidative stress biomarkers of streptozotocin-induced diabetic rats. Brazilian Journal of Medical and Biological Research, v.40, n.8, p.1095-9, 2007. SABU, M.C.; SMITHA, K.; KUTTAN, R. Anti-diabetic activity of green tea polyphenols and their role in reducing oxidative stress in experimental diabetes. Journal of Ethnopharmacology, v.83, n.1-2, p.109-16, 2002.

SABU, M.C.; KUTTAN, R. Antidiabetic activity of Aegle marmelos and its relationship with its antioxidant properties. Indian Journal of Physiology \& Pharmacology, v.48, n.1, p.81-8, 2004.

SAXENA, A.; VIKRAM, N. Role of selected Indian plants in the management of type 2 diabetes: A review. Journal of Alternative and Complementary Medicine, v.10, p.369-78, 2004.

SHARMA, R.; MISRA, A. Autoimmunity and gestational diabetes. The National Medical Journal of India, v.6, n.6, p.272-3, 1993.

SHIBANO, M. Antioxidant constituents in the dayflower (Commelina communis L.) and their alpha-glucosidaseinhibitory activity. Nature Medicine, v.62, p.3, p.349-53, 2008. SINZATO, Y.K. et al. Neonatally-Induced diabetes: Lipid profile outcomes and oxidative stress status in adult rats. Revista da Associação Médica Brasileira, v.55, n.4, p.3848, 2009.

SOUZA, M.S.S. et al. Effects of cigarette smoke exposure on pregnancy outcome and offspring of diabetic rats. Reproduction Biomedicine online, v.18, n.4, p.562-7, 2009. SUBA, V. et al. Anti-diabetic potential of Barleria lupulina extract in rats. Phytomedicine, v.11, p.202-5, 2004

TAKAKU, M. et al. Tipos de diabete. Femina, v.34, n.11, p.7636, 2006.

TROCINO, R.A. et al. Significance of glutathione depletion and oxidative stress in early embryogenesis in glucoseinduced rat embryo culture. Diabetes, v.44, n.8, p.992-8, 1995.

UGOCHUKWU, N.H.; COBOURNE, M.K. Modification of renal oxidative stress and lipid peroxidation in streptozotocin-induced diabetic rats treated with extracts from Gongronema latifolium leaves. Clinical Chimica Acta, v.336, n.1-2, p.73-81, 2003.

VENKATESWARAN, S.; PARI, L.; SARAVANAN, G. Effect of Phaseolus vulgaris on circulatory antioxidants and lipids in rats with streptozotocin-induced diabetes. Journal of Medical Food, v.5, n.2, p.97-103, 2002.

VENKATESWARAN, S.; PARI, L. Effect of Coccinia indica

Rev. Bras. Pl. Med., Botucatu, v.13, n.3, p.367-373, 2011. 
leaf extract on plasma antioxidants in streptozotocininduced experimental diabetes in rats. Phytotherapy Research, v.17, n.6, p.605-8, 2003.

VOLPATO, G.T. et al. Effect of Bauhinia forficata aqueous extract on the maternal-fetal outcome and oxidative stress biomarkers of streptozotocin-induced diabetic rats. Journal of Ethnopharmacology, v.116, n.1, p.131-7, 2008. VUKSAN, V.; SIEVENPIPER, J.L. Herbal remedies in the management of diabetes: lessons learned from the study of ginseng. Nutrition, Metabolism, and Cardiovascular Diseases, v.15, p.149-60, 2005.

WENTZEL, P.; WELSH, N.; ERIKSSON, U.J. Developmental damage, increased lipid peroxidation, diminished cyclooxygenase-2 gene expression, and lowered PGE2 levels in rat embryos exposed to a diabetic environment. Diabetes, v.48, p.813-20, 1999.

YANG, X.; BORG, L.A.H.; ERIKSSON, U.J. Altered mitochondrial morphology of rat embryos in diabetic pregnancy. Anatomical Record, v.241, p.255-67, 1995.

YANG, X.; BORG, L.A.H.; ERIKSSON, U.J. Altered metabolism and superoxide generation in neural tissue of rat embryos exposed to high glucose. American Journal of Physics, v.272, p.E173-80, 1997.

YEH, G. et al. Systematic review of herbs and dietary supplements for glycaemic control in diabetes. Diabetes Care, v.26, p.1277-94, 2003.

ZHANG, X.F.; TAN, B.K. Antihyperglycaemic and anti-oxidant properties of Andrographis paniculata in normal and diabetic rats. Clinical and Experimental Pharmacology and Physiology, v.27, n.5-6, p.358-63, 2000.

ZHAO, Z.; REECE, E.A. Experimental mechanisms of diabetic embryopathy and strategies for development therapeutic interventions. Journal of the Society for Gynecologic Investigation, v.12, p.549-57, 2005. 